\title{
Replik auf Leserbrief der Kollegen der Hanseklinik in Lübeck
}

Das Erfreuliche an Leserbriefen ist die Möglichkeit der Replik, in der im Artikel offensichtlich unzureichend präzise Dargestelltes präzisiert werden kann. Dass die wissenschaftliche Auseinandersetzung um das Lipödem bei den Lesern der Phlebologie auf großes Interesse stößt, zeigt auch die Rankingliste der „meistgelesenen“ Artikel dieser Zeitschrift, in der sowohl unsere Artikel über die Mythen des Lipödems als auch die Leserbriefe zu diesen Artikeln unter den Top Ten platziert sind [1]. Insofern danken wir den Autoren aus Lübeck für die Gelegenheit, ihre erwähnten Kritikpunkte aufzugreifen. Bemerkenswert erscheint uns in diesem Zusammenhang, dass zu unserer Artikelserie bislang nur Leserbriefe von die Liposuktion durchführenden Kollegen die Redaktion erreicht haben - nicht eine kritische Rückmeldung erhielten wir bisher von konservativen Behandlern.

Neben Zustimmung, die uns die Schreiber des Leserbriefs zollen, werden drei Aspekte unseres Artikels über die Liposuktion kritisch beurteilt:

1. unsere Zweifel an der - von Operateuren - regelhaft geäußerten, „langfristigen Wirksamkeit“ der Liposuktion

2. der von uns postulierte Placeboeffekt bei der Messung des Therapieerfolgs

3. bis zu welchem BMI adipösen Patienten eine Liposuktion empfohlen werden sollte

Zu 1: Im Gegensatz zur Wahrnehmung der Kollegen aus Lübeck zitierten wir nicht nur Baumgartner, der an die „langfristig wirksame Befund- und Beschwerdebesserung nach Liposuktion “ glaubt. Auf den Seiten 51 und 52 wurden auch die Kollegen Witte, Heck, Cornely, Schmeller und Rapprich mit ähnlichen Statements zitiert. Auch all diese, die Fettabsaugung durchführenden Kollegen, sind vom „dauerhaften Erfolg“ der Liposuktion überzeugt.

Allerdings macht auch die Veröffentlichung einer Studie im British Journal of Dermatology aus einem Glauben noch lange kein Faktum. Einige Stichpunkte mögen verdeutlichen, dass der Glaube an die „langfristige Beschwerdebesserung nach Liposuktion“ auf tönernen Füssen steht:
- Die verwendeten Fragebögen waren nicht validiert.

- Wie können Patienten selbst „Ödembildung“, „Hämatombildung“ bzw. „Einschränkung der Beweglichkeit“ beurteilen?

- Der für die Langzeitprognose essentielle Gewichtsverlauf der einzelnen Patientinnen wurde in beiden Studien nicht einmal erwähnt.

- Um zu nachzuweisen, ob das entfernte Fettgewebe tatsächlich langfristig bzw. dauerhaft entfernt wurde, hätte eine Volumetrie an den operierten Körperregionen vor und nach den Operationen durchgeführt werden müssen.

- Die Studie wurde nicht untersucherunabhängig, sondern durch die Operateure selbst vorgenommen, die damit ihre eigenen Operationsergebnisse beurteilen.

- Als „interne Vergleichsgruppe“ dient den Kollegen aus Lübeck die Komplexe physikalische Entstauungstherapie (KPE). Für diese Therapie - de facto die Kombination von Manueller Lymphdrainage und Kompression - fehlt ja selbst jeglicher Wirksamkeitsnachweis. Die Autoren vergleichen nun eine Therapieoption (Liposuktion) auf Basis einer völlig unzureichenden Studienlage [2, 3, 4] mit einer anderen Therapieoption (KPE), für die gar keine Studienlage existiert [5].

Ausdrücklich erwähnten wir in unserem Artikel, dass die Studien von Schmeller und Baumgartner - unter den existierenden Untersuchungen - die höchste Qualität aufweisen. Dennoch bleiben deutlich limitierende Faktoren, so dass die Aussage, „Liposuktion wirke langfristig“, weiterhin eher dem Bereich der Mythen als dem der Fakten zuzuordnen ist. Gerade in Zeiten, in denen Lobbyismus und politischer Populismus die Überprüfung medizinscher Verfahren mittels wissenschaftlicher Evidenz auszuhöhlen drohen, ist das Beharren auf sauberen, untersucher- und damit interessenunabhängigen Studien essentiell. Bestrebungen eines Jens Spahn, Therapien wie die Liposuktion per Ermächtigung zur Kassenleistung zu machen, obwohl, so auch Spahn selbst, „der Nutzen noch nicht hinreichend belegt “ [6] ist, wäre eine Abkehr von Entscheidungen aufgrund evidenzbasierter Kriterien - und gefährdet die Gesundheit unserer Patienten.

Zu 2: Zum Placeboeffekt bemerken die Lübecker Kollegen: „Man spricht von einem Placeboeffekt, wenn keine nachweisbar wirksame Therapiemaßnahme durchgeführt wurde und trotzdem eine Wirkung festgestellt wurde.“ Diese Feststellung ist richtig - aber bei weitem nicht vollständig. Placebo-Wirkung erklärt auch jenen Teil am Therapieergebnis, der nicht auf die Behandlung selbst zurückzuführen ist [7].

Jeder Therapieerfolg setzt sich grundsätzlich aus drei Teilen zusammen: dem Effekt des natürlichen Krankheitsverlaufs, dem Placeboeffekt (z. B. der Wirkung der Erwartungen) und natürlich dem Effekt der Therapie selbst (der hoffentlich möglichst groß ist). Dabei ist die Placebo-Wirkung besser als ihr Ruf. Oft handelt es sich um Erwartungen, die durch das ärztliche Gespräch erzeugt werden. Dass die mit einer Behandlung verbundene Erwartungshaltung einen großen Einfluss auf den Erfolg einer Therapie hat, ist bekannt [7]. Wir stimmen den Autoren des Leserbriefes zu: Dies gilt für alle Therapiemaßnahmen, konservative wie operative - und daher auch für die Liposuktion!

Daher sind Kontrollgruppendesigns zwingend notwendig, um herauszufinden, wie groß der Effekt der Liposuktion selbst ist. Dass, wie die Kollegen der Hanseklinik schreiben, ein „Effekt postoperativ regelhaft auftritt“, ist aus methodischer Sicht in keiner Weise ausreichend, um die Wirkung „kausal“ allein der Liposuktion zuzuschreiben. Der Zeitraum mit KPE vor einer Liposuktion („interne Vergleichsgruppe“) ist sicher keine geeignete Kontrollgruppe, da wie oben dargelegt, für die Therapie dieser Kontrollgruppe ebenfalls keine Evidenz vorliegt.

Auch wir denken nicht, dass die Patientinnen „den Operateuren nach dem Mund reden“. Fakt ist aber, dass die Patientinnen selbst durch Informationen aus den Medien, dem Vergleich mit dem gängigen 
Schönheitsideal - mit der enormen Idealisierung schlanker Beine - einen hohen Erwartungsdruck aufbauen. Eine Ahnung dieses enormen Erwartungsdrucks erhält man, wenn man das Stichwort „Liposuktion“ bei YouTube oder in den einschlägigen Lipödem-Foren eingibt. Bei Patienten, die mit der Liposuktion unzufrieden sind, kann sich eine kognitive Dissonanz entwickeln. Diese tritt dann auf, wenn man große Anstrengungen (zeitliche oder finanzielle) auf sich genommen hat, nur um dann festzustellen, dass das Ergebnis den Erwartungen nicht gerecht wird. Da die kognitive Dissonanz als unangenehm empfunden wird, versuchen die Patientinnen die dissonanten Kognitionen in Einklang zu bringen, um den negativen Gefühlszustand zu beenden - mit der Folge, die Ergebnisse oft besser zu bewerten [8]!

Zu 3: Wir freuen uns über die Zustimmung, dass Lipödem und Adipositas sauber getrennt werden müssen. Die Kollegen aus Lübeck betonen auch völlig korrekt, dass beide Entitäten „oft“ (präziser wäre hier allerdings „meistens“ [9]) gemeinsam auftreten.

Nicht korrekt ist der im Leserbrief - angeblich von uns genannte - BMI von $32 \mathrm{~kg} /$ $\mathrm{m}^{2}$, bei dem spätestens eine abdominelle Adipositas vorliegt. Hier hatten wir einen BMI von $35 \mathrm{~kg} / \mathrm{m}^{2}$ genannt, im gleichen Satz aber auch darauf hingewiesen, dass die Waist-to-Height Ratio häufig der aussagekräftigere Parameter ist (Seite 52). Dennoch ist der von uns genannte BMI von $35 \mathrm{~kg} / \mathrm{m}^{2}$ tatsächlich zu starr. Wir stimmen mit den Autoren des Leserbriefes überein, dass es - seltene - Ausnahmen dieser BMIObergrenze geben kann. Die von den Lübecker Kollegen gezeigte Patientin in $>$ Abb. 1 (quasi das selten vorkommende „Idealbild“ einer Lipödempatientin) ist eine dieser Ausnahmen, der wir - die im Artikel unter „Abschließende Einschätzung“ genannten Bedingungen vorausgesetzt - auch eine Liposuktion empfohlen hätten. Und würde diese Patientin in Norddeutschland wohnen, so hätten wir auch ganz sicher - aufgrund der dort auf hohem medizinischem Niveau durchgeführten Liposuktionen - die Operateure der Hanseklinik in Lübeck für diesen Eingriff empfohlen.
Entschieden anderer Meinung als die Leserbriefautoren sind wir jedoch bei der Patientin in > Abb. 2. Diese Patientin mit einem BMI von $41 \mathrm{~kg} / \mathrm{m}^{2}$ hat - ganz offensichtlich - auch eine abdominelle Adipositas. Abhängig vom Leidensdruck der Patientin sowie weiteren Adipositas-assoziierten Begleiterkrankungen (z. B. arterielle Hypertonie, Diabetes mellitus oder Gonarthrose) hätten wir bei dieser Patientin sicher eine bariatrische OP erwogen.

Da chirurgisch tätige Ärzten dazu verpflichtet sind, Patientinnen über alle Behandlungsmöglichkeiten bzw. über Behandlungsalternativen vor den Operationen aufzuklären, gehen wir davon aus, dass auch die in $>$ Abb. 2 gezeigte Patientin, mit einem BMI von über $40 \mathrm{~kg} / \mathrm{m}^{2}$, entsprechend der Leitlinien [10] von den Lübecker Kollegen über die Möglichkeit eines adipositaschirurgischen Eingriffes aufgeklärt wurde - die Patientin sich aber dennoch für die Liposuktion entschied.

Von einer von uns favorisierten bariatrischen OP und die hierdurch mögliche - langfristige - Gewichtsreduktion hätte diese Patientin allerdings nicht nur dramatisch hinsichtlich ihres Lipödems profitiert, auch die schon bestehenden (bzw. bei diesem BMI drohenden!) Adipositas-assoziierten Begleiterkrankungen hätten eine deutliche Besserung, oft auch Remission erfahren - ganz abgesehen von der Verlängerung der Lebensspanne der Patientin. Um den Einfluss der bariatrischen OP auf das Lipödem nachzuweisen, führen wir gerade gemeinsam mit den Kollegen der Klinik für Allgemein- und Viszeralchirurgie am Universitätsklinikum Freiburg eine Studie durch.

Der von den Leserbriefautoren suggerierte - ursächliche - Zusammenhang zwischen der Liposuktion und der nachfolgenden Gewichtsabnahme der initial morbid adipösen Patientin mag im Einzelfall vorkommen, widerspricht aber sicher der Erfahrung der großen Mehrheit konservativ tätiger Behandler. Vielmehr neigt gerade dieses Patientengut zu „Jo-Jo-Erfahrungen“, so dass der initiale Erfolg der Liposuktion nur eine kurze Haltbarkeit aufweist. Wir sind in Teil 4 unserer Artikelserie ausführlich auf diesen Punkt eingegangen.
Selbst in der von den Lübecker Kollegen hervorgehobenen British Journal of Dermatology-Publikation von Baumgartner werden diese typischen Gewichtsschwankungen von Lipödem-Patientinnen deutlich. In > Fig. 10 werden mehrere Bilder einer Lipödempatientin vor und nach Liposuktion gezeigt. Die Patientin hat im Untersuchungszeitraum erhebliche Gewichtschwankungen erlebt, allein zwischen 2010 und 2014 einen Gewichtsanstieg von $72 \mathrm{~kg}$ auf $88 \mathrm{~kg}$. Baumgartner et al. kommentieren dies wie folgt: „Despite an increase in weight and in volume on the trunk, the morphology of the legs is unchanged“ [11]. Die Lübecker Kollegen ignorieren hierbei die Bedeutung der Zunahme an vorwiegend viszeralem Fett in der Abdominalregion, was bekanntlich mit einem erhöhten kardiovaskulären Risiko einhergeht. Dieses nach Liposuktion beobachtbare Phänomen wurde bereits in der - in den USA vielbeachteten - prospektiven, randomisiert-kontrollierten Studie der Kollegen der University of Colorado bestätigt [12].

Dieser divergente Blick erklärt sich am ehesten durch den Unterschied zwischen der fokussiert dermatologisch-chirurgischen Sichtweise der Leserbriefautoren auf die Patientin und dem internistischen Blick des Erstautors der Artikelserie.

Angesichts der zustimmenden Äußerungen der Lübecker Kollegen erscheinen uns bei Gesamtbetrachtung die divergenten Standpunkte überschaubar. So betonen die Leserbriefautoren gleich zu Beginn: „Die abschließend aufgeführten Kriterien für den Einsatz der Liposuktion halten wir für korrekt. “ Diese dort in Teil 4 unserer Artikelserie aufgeführten Kriterien („Abschließende Einschätzung “) entsprechen weitgehend dem Consensus, der auf dem $2^{\text {nd }}$ European Lipoedema Forum verabschiedet wurde. Hier trafen sich im März dieses Jahres renommierte Lipödemexperten aus sieben europäischen Ländern in Hamburg, um gemeinsam diagnostische Kriterien des Lipödems sowie ein therapeutisches Konzept zu erarbeiten. Die Ergebnisse dieser Konferenz kommen einem Paradigmenwechsel des Lipödems gleich und werden Gegenstand im fünften und letzten Teil unserer Artikelserie über die Mythen und Fakten des Lipödems sein. 
Leserbriefe stellen nicht unbedingt die Meinung von Herausgebern oder Verlag dar. Herausgeber und Verlag behalten sich vor, Leserbriefe nicht, gekürzt oder in Auszügen zu veröffentlichen.

\section{Interessenkonflikt}

Die Autoren geben an, dass kein Interessenkonflikt besteht.

\section{Autorinnen/Autoren}

Tobias Bertsch

Nestor Torio-Padron

Gabriele Erbacher

\section{Korrespondenzadresse}

Dr. Tobias Bertsch

Földiklinik Hinterzarten - Europäisches Zentrum für Lymphologie

E-Mail: tobias.bertsch@foeldiklinik.de
[1] Meistgelesene Artikel in der Phlebologie: Abrufbar unter: https://www.thiemeconnect.de/products/ejournals/topten/ $10.1055 / \mathrm{s}-00034913$ (Stand 02. April 2019)

[2] Gemeinsamer Bundesausschuss vom 20. Juli 2017. Tragende Gründe zum Beschluss des Gemeinsamen Bundesausschusses über eine Änderung der Richtlinie Methoden Krankenhausbehandlung: Liposuktion bei Lipödem. Abrufbar unter: https://www.g-ba.de/downloads/40-268-4488/2017-07-20_KHMe-RL_ Liposuktion_TrG.pdf

[3] Ärzteblatt.de. Liposuktion bei Lipödem: Bundessozialgericht weist Klage auf Kostenerstattung ab. Abrufbar unter: https:// www.aerzteblatt.de/nachrichten/94709/ Liposuktion-bei-Lipoedem-Bundessozialgericht-weist-Klage-auf-Kostenerstattung-ab

[4] Bertsch T, Erbacher G. Torio-Padron, N. Lipödem - Mythen und Fakten Teil 4. Phlebologie 2019; 48: 47-56

[5] Bertsch T, Erbacher G. Lipödem - Mythen und Fakten Teil 2. Phlebologie 2018; 47: 120-126

[6] Ärzteblatt online. Abrufbar unter: https:// www.aerzteblatt.de/nachrichten/100286/ Spahn-will-Liposuktion-zur-Kassenleistungmachen

[7] Evers AWM, Colloca L, Blease C et al. Implications of Placebo and Nocebo Effects for
Clinical Practice: Expert Consensus. Psychother Psychosom 2018; 87(4): 204-210

[8] Irle M. (Hrsg): Festinger L.: Theorie der Kognitiven Dissonanz. Bern: Huber 2012. Unveränderter Nachdruck der Ausgabe von 1978 Hans Huber

[9] Bertsch T, Erbacher G. Lipödem - Mythen und Fakten Teil 3. Phlebologie 2018; 47: 188-197

[10] S3-Leitlinie: Chirurgie der Adipositas und metabolischer Erkrankungen. Abrufbar unter: https://www.awmf.org/uploads/tx_ szleitlinien/088-001I_S3_Chirurgie-Adipositas-metabolische-Erkrankugen_2018-02. pdf

[11] Baumgartner A, Hueppe M, Schmeller W. Long-term benefit of liposuction in patients with lipoedema: a follow-up study after an average of 4 and 8 years. $\mathrm{Br}$ ] Dermato 2016; 174: 1061-7

[12] Hernandez TL, Eckel RH et al. Fat redistribution following suction lipectomy: defense of body fat and patterns of restoration. Obesity 2011; 19(7): 1388-95

\section{Bibliografie}

DOI https://doi.org/10.1055/a-0889-4845 Phlebologie 2019; 48: 199-201

(c) Georg Thieme Verlag KG Stuttgart · New York ISSN 0939-978X 